\title{
OSCILLATION CRITERIA FOR NONLINEAR INHOMOGENEOUS HYPERBOLIC EQUATIONS WITH DISTRIBUTED DEVIATING ARGUMENTS ${ }^{1}$
}

\author{
XINZHI LIU and XILIN FU ${ }^{2}$ \\ University of Waterloo \\ Department of Applied Mathematics \\ Waterloo, Ontario, Canada N2L $3 G 1$
}

(Received March, 1995; Revised July, 1995)

\begin{abstract}
This paper investigates the oscillatory properties of solutions of nonlinear inhomogeneous hyperbolic equations with distributed deviating arguments subject to two different boundary conditions. Several oscillation criteria are establishing employing Green's Theorem and certain differential inequalities. An example is also given.
\end{abstract}

Key words: Oscillation, Hyperbolic Equation with Deviating Arguments, Inhomogeneous.

AMS (MOS) subject classifications: $35 \mathrm{~B} 05,34 \mathrm{~K} 40,34 \mathrm{~A} 40$.

\section{Introduction}

The oscillation theory of hyperbolic partial differential equations with delays has become an important area of investigation in the past few years, see [1]-[4] and references therein. Recently, some interesting oscillation conditions have been obtained in [1] for homogeneous hyperbolic equations with distributed deviating arguments. In this paper, we shall consider the following nonlinear inhomogeneous hyperbolic equation with distributed deviating arguments:

$$
\begin{gathered}
\frac{\partial^{2}}{\partial t^{2}}[u+\lambda(t) u(x, t-\tau)]+\int_{a}^{b} p(x, t, \xi) F(u(x, g[t, \xi)]) d \sigma(\xi) \\
=a(t) \Delta u+f(x, t), \quad(x, t) \in G,
\end{gathered}
$$

where $G=\Omega \times R_{+}, \Omega$ is a bounded domain in $R^{n}$ with piecewise smooth boundary $\partial \Omega, R_{+}=$ $[0,+\infty), u=u(x, t), \Delta$ is the Laplacian in $R^{n}, p \in C\left[\bar{\Omega} \times R_{+} \times J, R_{+}\right], J=[a, b], F \in C[R, R]$, $a \in C\left[R_{+}, R_{+}\right], \lambda \in C^{2}\left[R_{+}, R\right], \tau$ is a constant, $f \in C\left[\Omega \times R_{+}, R\right], g \in C\left[R_{+} \times J, R\right], \sigma \in$ $[J, R]$, and the integral in $(E)$ is a Stieltjes integral. Throughout this paper we assume that $g(t, \xi)$ is nondecreasing in $t$ and $\xi$ respectively, with $g(t, \xi)<t$ for any $\xi$ and $\lim _{t \rightarrow+\infty} \inf _{\xi \in J} g(t, \xi)=$ $+\infty$, and that $\sigma(\xi)$ is nondecreasing in $\xi$. We shall consider two kinds of boundary conditions:

\footnotetext{
${ }^{1}$ Research supported by NSERC-Canada.

${ }^{2}$ On leave from Shandong Normal University. 


$$
\frac{\partial u}{\partial N}+\gamma(x, t) u=\mu(x, t),(x, t) \in \partial \Omega \times R_{+}
$$

and

$$
u=\phi(x, t),(x, t) \in \partial \Omega \times R_{+}
$$

where $N$ is the unit outnormal vector to $\partial \Omega, \gamma \in C\left[\partial \Omega \times R_{+}, R_{+}\right], \mu, \phi \in C\left[\partial \Omega \times R_{+}, R\right]$.

A solution $u(x, t)$ of equation $(E)$ satisfying certain boundary conditions is said to be oscillatory if, for any positive number $\alpha$ there exists a point $\left(x_{0}, t_{0}\right) \in \Omega \times[\alpha,+\infty)$ such that $u\left(x_{0}, t_{0}\right)=0$.

The objective of this paper is to study the oscillatory properties of solutions of equation $(E)$ subject to boundary conditions $(B 1)$ and $(B 2)$. In Section 2, we shall establish several oscillation criteria for boundary value problems $(E)-(B 1)$ and $(E)-(B 2)$, employing Green's Theorem and certain differential inequalities. We shall then develop, in Section 3 , some results on differential inequalities which, in addition to their independent value, enable us to obtain (in Section 4) further oscillation criteria regarding boundary value problems $(E)-(B 1)$ and $(E)-(B 2)$. An example is also given.

\section{Oscillation Criteria}

Let us begin with listing the following notations and assumptions.

$$
\begin{gathered}
U(t)=\frac{1}{|\Omega|} \int_{\Omega} u(x, t) d x, \text { where }|\Omega|=\int_{\Omega} d x, \\
P(t, \xi)=\min _{x \in \bar{\Omega}} p(x, t, \xi), \\
\tilde{\mu}(t)=\frac{1}{|\Omega|} \int_{\partial \Omega} \mu(x, t) d S, \\
\tilde{f}(t)=\frac{1}{|\Omega|} \int_{\Omega} f(x, t) d x, \\
R_{1}(t)=\tilde{\mu}(t)+\tilde{f}(t),
\end{gathered}
$$

and

$$
F(u) \text { is convex in } R_{+} \text {and }-F(-u)=F(u)>0, u \in R_{+},
$$

where $d S$ is the surface integral element on $\partial \Omega$.

Theorem 1: Assume that condition (6) holds. If the differential inequalities with distributed deviating arguments

$$
\frac{d^{2}}{d t^{2}}[U(t)+\lambda(t) U(t-\tau)]+\int_{a}^{b} P(t, \xi) F(U[g(t, \xi)]) d \sigma(\xi) \leq R_{1}(t)
$$




$$
\frac{d^{2}}{d t^{2}}[U(t)+\lambda(t) U(t-\tau)]+\int_{a}^{b} P(t, \xi) F(U[g(t, \xi)]) d \sigma(\xi) \leq-R_{1}(t)
$$

have no eventually positive solutions, then all solutions of problem $(E)-(B 1)$ are oscillatory in $G$.

Proof: Let $u(x, t)$ be a nonoscillatory solution of problem $(E)-(B 1)$. We may assume that $u(x, t)>0$ for $(x, t) \in \Omega \times[\alpha,+\infty)$, where $\alpha$ is a positive number. Since $\lim _{t \rightarrow+\infty}$ $\min _{\xi \in J}\{g(t, \xi)\}=+\infty$, it follows that there exists a $t_{0} \geq \alpha$ such that

$$
u(x, t-\tau)>0 \text { and } u[x, g(t, \xi)]>0, t>t_{0}, \xi \in J .
$$

Integrating both sides of equation $(E)$ with respect to $x$ over domain $\Omega$ we obtain

It is easy to see that

$$
\begin{gathered}
\left.\frac{d^{2}\left[t ^ { 2 } \left[\int_{\Omega} u(x, t) d x+\right.\right.}{}+\lambda(t) \int_{\Omega} u(x, t-\tau) d x\right]+\int_{\Omega} \int_{a}^{b} p(x, t, \xi) F(u[x, g(t, \xi)]) d \sigma(\xi) d x \\
=a(t) \int_{\Omega} \Delta u(x, t) d x+\int_{\Omega} f(x, t) d x, \quad t \geq t_{0} .
\end{gathered}
$$

$$
\int_{\Omega} \int_{a}^{b} p(x, t, \xi) F(u[x, g(t, \xi)]) d \sigma(\xi) d x=\int_{a}^{b} \int_{\Omega} p(x, t, \xi) F(u[x, g(t, \xi)]) d x d \sigma(\xi) .
$$

Using Green's Theorem, we have

$$
\int_{\Omega} \Delta u(x, t) d x=\int_{\partial \Omega} \frac{\partial u}{\partial N} d S=\int_{\partial \Omega}[\mu(x, t)-\gamma(x, t) u(x, t)] d S \leq \int_{\partial \Omega} \mu(x, t) d S, t \geq t_{0} .
$$

In view of condition (6), it follows from Jensen's inequality that

$$
\int_{\Omega} F(u[x, g(t, \xi)]) d x \geq|\Omega| F\left(\frac{1}{|\Omega|} \int_{\Omega} u[x, g(t, \xi)] d x\right), t \geq t_{0} .
$$

Combining (8)-(11) we have

$$
\begin{gathered}
\frac{d^{2}}{d t^{2}}\left[\int_{\Omega} u(x, t) d x+\lambda(t) \int_{\Omega} u(x, t-\tau) d x\right] \leq a(t) \int_{\partial \Omega} \mu(x, t) d S \\
+\int_{\Omega} f(x, t) d x-|\Omega| \int_{a}^{b} \min _{x \in \bar{\Omega}} p(x, t, \xi) F\left(\frac{1}{|\Omega|} \int_{\Omega} u[x, g(t, \xi)] d x\right) d \sigma(\xi), t \geq t_{0} .
\end{gathered}
$$

Thus we can see that the function defined by (1) is a positive solution of inequality (I1); but this contradicts the condition of the theorem.

If $u(x, t)<0$ for $(x, t) \in \Omega \times[\alpha,+\infty)$, then set

$$
u^{*}(x, t)=-u(x, t), \quad(x, t) \in \Omega \times[\alpha,+\infty) .
$$

Using $F(-u)=-F(u)$, for $u \in R_{+}$, it is easy to check that $u^{*}(x, t)$ is a positive solution of the problem: 


$$
\left\{\begin{array}{l}
\frac{\partial^{2}}{\partial t^{2}}[u+\lambda(t) u(x, t-\tau)]+\int_{a}^{b} p(x, t, \xi) F(u[x, g(t, \xi)]) d \sigma(\xi)=a(t) \Delta u-f(x, t),(x, t) \in G \\
\frac{\partial u}{\partial N}+\gamma(x, t) u=-\mu(x, t),(x, t) \in \partial \Omega \times[0,+\infty)
\end{array}\right.
$$

and it satisfies

$$
\begin{gathered}
\frac{d^{2}}{d t^{2}}\left[\int_{\Omega} u^{*}(x, t) d x+\lambda(t) \int_{\Omega} u^{*}(x, t-\tau) d x\right] \\
+|\Omega| \int_{a}^{b} \min _{x \in \bar{\Omega}} p(x, t, \xi) F\left(\frac{1}{|\Omega|} \int_{\Omega} u^{*}[x, g(t, \xi)] d x\right) d \sigma(\xi) \\
\leq-a(t) \int_{\partial \Omega} \mu(x, t) d S-\int_{\Omega} f(x, t) d x, \quad t \geq t_{0} .
\end{gathered}
$$

Thus it follows that the function

$$
U^{*}(t)=\frac{1}{|\Omega|} \int_{\Omega} u^{*}(x, t) d x
$$

is a positive solution of inequality (I2) for $t \geq t_{0}$ which also contradicts the assumption of the theorem. This completes the proof of Theorem 1 .

The following fact and notations shall be used later in the proof of Theorem 2. Consider the Dirichlet problem

$$
\left\{\begin{array}{c}
\Delta u+\lambda u=0 \text { in } \Omega \\
\left.u\right|_{\partial \Omega}=0
\end{array}\right.
$$

where $\lambda=$ constant. It is well-known [3] that the smallest eigenvalue $\lambda_{0}$ and the corresponding eigenfunction $\Phi(x)$ are positive. We define

$$
\begin{gathered}
R_{2}(t)=\left[-a(t) \int_{\partial \Omega} \phi(x, t) \frac{\partial}{\partial N} \Phi(x) d S+\int_{\Omega} f(x, t) \Phi(x) d x\right] \frac{1}{\int_{\Omega} \Phi(x) d x}, \\
V(t)=\frac{1}{\int_{\Omega} \Phi(x) d x} \int_{\Omega} u(x, t) \Phi(x) d x
\end{gathered}
$$

where $u(x, t)$ is a solution of the problem $(E)-(B 2)$.

Theorem 2: Assume that condition (6) holds. If the differential inequalities with distributed deviating arguments

$$
\begin{aligned}
& \frac{d^{2}}{d t^{2}}[V(t)+\lambda(t) V(t-\tau)]+\lambda_{0} a(t) V(t)+\int_{a}^{b} P(t, \xi) F(V[g(t, \xi)]) d \sigma(\xi) \leq R_{2}(t), \\
& \left.\frac{d^{2}}{d t^{2}} V(t)+\lambda(t) V(t-\tau)\right]+\lambda_{0} a(t) V(t)+\int_{a}^{b} P(t, \xi) F\left(V[(g(t, \xi)]) d \sigma(\xi) \leq-R_{2}(t)\right.
\end{aligned}
$$


have no eventually positive solutions, then all solutions of problem $(E)-(B 2)$ are oscillatory in $G$.

Proof: Let $u(x, t)$ be a solution of problem $(E)-(B 2)$ having no zeros in the domain $\Omega \times[\alpha,+\infty)$ for some $\alpha>0$. If $u(x, t)>0$ for $(x, t) \in \Omega \times[\alpha,+\infty)$, then there exists a $t_{0}>\alpha$ such that

$$
u(x, t-\tau)>0 \text { and } u[x, g(t, \xi)]>0, t>t_{0}, \xi \in J .
$$

Multiplying both sides of $(E)$ by the eigenfunction $\Phi(x)$ and integrating with respect to $x$ over the domain $\Omega$, we get

$$
\begin{aligned}
& \frac{d^{2}}{d t^{2}}\left[\int_{\Omega} u(x, t) \Phi(x) d x+\lambda(t) \int_{\Omega} u(x, t-\tau) \Phi(x) d x\right] \\
& \quad+\int_{\Omega} \int_{a}^{b} p(x, t, \xi) F(u[x, g(t, \xi)]) \Phi(x) d \sigma(\xi) d x \\
& =a(t) \int_{\Omega} \Delta u(x, t) \Phi(x) d x+\int_{\Omega} f(x, t) \Phi(x) d x, t \geq t_{0} .
\end{aligned}
$$

From Green's Theorem, it follows that

$$
\begin{gathered}
\int_{\Omega} \Delta u(x, t) \Phi(x) d x=\int_{\partial \Omega}\left[\Phi(x) \frac{\partial}{\partial N} u(x, t)-u(x, t) \frac{\partial}{\partial N} \Phi(x)\right] d S+\int_{\Omega} u(x, t) \Delta \Phi(x) d x \\
=-\int_{\partial \Omega} \phi(x, t) \frac{\partial}{\partial N} \Phi(x) d S-\lambda_{0} \int_{\Omega} u(x, t) \Phi(x) d x, \quad t \geq t_{0} .
\end{gathered}
$$

Using Jensen's inequality, we obtain

$$
\int_{\Omega} F(u[x, g(t, \xi)]) \Phi(x) d x \geq \int_{\Omega} \Phi(x) d x \cdot F\left(\frac{1}{\int_{\Omega} \Phi(x) d x} \int_{\Omega} u[x, g(t, \xi)] \Phi(x) d x\right) .
$$

Combining (15)-(17), we get

$$
\begin{gathered}
\frac{d^{2}}{d t^{2}}\left[\int_{\Omega} u(x, t) \Phi(x) d x+\lambda(t) \int_{\Omega} u(x, t-\tau) \Phi(x) d x\right] \\
+\left[\int_{\Omega} \Phi(x) d x\right] \cdot \int_{a}^{b} \min _{x \in \bar{\Omega}} p(x, t, \xi) \cdot F\left(\frac{1}{\int_{\Omega} \Phi(x) d x} \int_{\Omega} u[x g(t, \xi)] \Phi(x) d x\right) d \sigma(\xi) \\
\leq-a(t) \int_{\partial \Omega} \phi(x, t) \frac{\partial}{\partial N} \Phi(x) d S-\lambda_{0} a(t) \int_{\Omega} u(x, t) \Phi(x) d x+\int_{\Omega} f(x, t) \Phi(x) d x, \quad t \geq t_{0} .
\end{gathered}
$$

Thus we see that the function defined by (14) is a positive solution of the inequality (I3) which contradicts the condition of the theorem.

If $u(x, t)<0$ for $(x, t) \in \Omega \times[\alpha,+\infty)$, then the function $u^{*}=-u$ is a positive solution of the problem 


$$
\left\{\begin{array}{l}
\left.\frac{\partial^{2}}{\partial t^{2}} u+\lambda(t) u(x, t-\tau)\right]+\int_{a}^{b} p(x, t, \xi) F(u[x, g(t, \xi)]) d \sigma(\xi)=a(t) \Delta u-f(x, t),(x, t) \in G \\
u=-\phi(x, t), \quad(x, t) \in \partial \Omega \times[0,+\infty)
\end{array}\right.
$$

and it satisfies

$$
\begin{gathered}
\frac{d^{2}}{d t^{2}}\left[\int_{\Omega} u^{*} \Phi(x) d x+\lambda(t) \int_{\Omega} u^{*}(x, t-\tau) \Phi(x) d x\right] \\
+\left[\int_{\Omega} \Phi(x) d x\right] \cdot \int_{a}^{b} \min _{x \in \bar{\Omega}} p(x, t, \xi) F\left(\frac{1}{\int_{\Omega} \Phi(x) d x} \int_{\Omega} u^{*}[x, g(t, \xi)] \Phi(x) d x\right) d \sigma(\xi) \\
\leq-a(t) \int_{\partial \Omega}[-\phi(x, t)] \frac{\partial}{\partial N} \Phi(x) d S-\lambda_{0} a(t) \int_{\Omega} u^{*}(x, t) \Phi(x) d x+\int_{\Omega}[-f(x, t)] \Phi(x) d x, t \geq t_{0} .
\end{gathered}
$$

In other words, the function,

$$
V^{*}(t)=\frac{1}{\int_{\Omega} \Phi(x) d x} \int_{\Omega} u^{*}(x, t) \Phi(x) d x
$$

is a positive solution of inequality (I4) for $t \geq t_{0}$. This provides a contradiction. Thus the proof of Theorem 2 is complete.

\section{Delay Differential Inequalities}

From the discussion in Section 2, it follows that the problem of establishing oscillation criteria for $(E)$ can be reduced to the investigation of the properties of the solutions of delay inhomogeneous differential inequalities of the form

$$
\left.\frac{d^{2}}{d t^{2}} y(t)+\lambda(t) y(t-\tau)\right]+\int_{a}^{b} P(t, \xi) F(y[g(t, \xi)]) d \sigma(\xi) \leq R(t), \quad t \geq t_{0}
$$

where $\lambda \in C\left[\left[t_{0},+\infty\right), R\right], P \in C\left[\left[t_{0},+\infty\right) \times J, R_{+}\right], F \in C[R, R], R \in C\left[\left[t_{0},+\infty\right), R\right]$.

Theorem 3: Let $\lambda(t) \geq 0$ for $t \geq t_{0}>0$ and $F(y)>0$ for $y \in R_{+}$. If

$$
\liminf _{t \rightarrow+\infty} \int_{t_{1}}^{t}\left(1-\frac{s}{t}\right) R(s) d s=-\infty
$$

for every sufficiently large $t_{1}$, then the inhomogeneous differential inequality (I5) has no eventually positive solutions.

Proof: Suppose that $y(t)$ is an eventually positive solution of $(I 5)$. Then there exists a $t_{1}>t_{0}$ such that

$$
y(t)>0, y(t-\tau)>0 \text { and } y[g(t, \xi)]>0, t \geq t_{1}, \quad \xi \in J
$$


Hence we obtain

$$
\begin{aligned}
\left.\frac{d^{2}}{d t^{2}} y(t)+\lambda(t) y(t-\tau)\right] & \leq R(t)-\int_{a}^{b} P(t, \xi) F(y[g(t, \xi)]) d \sigma(\xi) \\
& \leq R(t), \quad t \geq t_{1} .
\end{aligned}
$$

Integrating the above inequality twice over $\left[t_{1}, t\right], t>t_{1}$, we get

where $c_{1}$ and $c_{2}$ are constants. Note that

$$
y(t)+\lambda(t) y(t-\tau) \leq c_{1}+c_{2}\left(t-t_{1}\right)+\int_{t_{1}}^{t} \int_{t_{1}}^{\eta} R(s) d s d \eta
$$

$$
\int_{t_{1}}^{t} \int_{t_{1}}^{\eta} R(s) d s d \eta=\int_{t_{1}}^{t}(t-s) R(s) d s
$$

Dividing both sides of the last inequality above by $t\left(t>t_{1}\right)$, we have

Using (18), we get

$$
\frac{1}{t}[y(t)-\lambda(t) y(t-\tau)] \leq \frac{c_{1}}{t}+c_{2}\left(1-\frac{t_{1}}{t}\right)+\int_{t_{1}}^{t}\left(1-\frac{s}{t}\right) R(s) d s .
$$

$$
\liminf _{t \rightarrow+\infty} \frac{1}{t}[y(t)+\lambda(t) y(t-\tau)]=-\infty .
$$

On the other hand, since $\lambda(t) \geq 0, y(t)>0$ and $y(t-\tau)>0$ for $t \geq t_{1}$, we have

$$
\liminf _{t \rightarrow+\infty} \frac{1}{t}[y(t)+\lambda(t) y(t-\tau)] \geq 0
$$

which contradicts (19). This completes the proof.

When $f(x, t) \equiv 0, \mu(x, t) \equiv 0$ and $\phi(x, t) \equiv 0$, the problem of establishing oscillation criteria for $(E)$ can be reduced to the investigation of the properties of the solutions of delay homogeneous differential inequalities of the forms

$$
\frac{d^{2}}{d t^{2}}[y(t)+\lambda(t) y h(t-\tau)]+\int_{a}^{b} P(t, \xi) F(y[g(t, \xi)]) d \sigma(\xi) \geq 0, \quad t \leq t_{0}
$$

and

$$
\frac{d^{2}}{d t^{2}}[y(t)+\lambda(t) y(t-\tau)]+\int_{a}^{b} P(t, \xi) F(y[g(t, \xi)]) d \sigma(\xi) \geq 0, \quad t \geq t_{0} .
$$

Along with $(I 6),(I 7)$ we consider the delay differential equation

$$
\frac{d^{2}}{d t^{2}}[y(t)+\lambda(t) y(t-\tau)]+\int_{a}^{b} P(t, \xi) F(y[g(t, \xi)]) d \sigma(\xi)=0, \quad t \geq t_{0},
$$

where $\lambda \in C\left[\left[t_{0}+\infty\right), R\right], P \in C\left[\left[t_{0},+\infty\right) \times J, R\right], F \in C[R, R]$.

Lemma 1: [5] Assume that

(I) $y \in C\left[\left[t_{0},+\infty\right), R_{+}\right]$ 
(II) $0<\lambda_{1} \leq \lambda(t) \leq \lambda_{2}, \quad t \geq t_{0}$, where $\lambda_{1}$, and $\lambda_{2}$ are constants;

(III) $y(t)+\lambda(t) y(t-\tau) \geq k_{1}, t \geq t_{0}, t_{0}, k_{1}>0$.

Then there exists a closed and measurable set $E \subset\left[t_{0},+\infty\right)$ and a constant $k>0$ such that

$$
y(t) \geq k, t \in E
$$

and

$$
\text { meas }(E \cap[t, t+2 \tau]) \geq \tau, \quad t \geq t_{0} .
$$

Theorem 4: Assume that $0<\lambda_{1} \leq \lambda(t) \leq \lambda_{2}, t \geq t_{0}$, that $-F(-y)=F(y)>0, y \in R_{+}$, and that

$$
F(y) \text { is a monotone increasing function in } R_{+} \text {. }
$$

If for any closed and measurable set $E \subset\left[t_{0},+\infty\right)$ for which meas $(E \cap[t, t+2 \tau]) \geq \tau, t \geq t_{0}$, the following condition holds

then

$$
\int_{E} \int_{a}^{b} P(s, \xi) d \sigma(\xi) d s=+\infty
$$

(i) the inequality (I6) has no eventually positive solutions;

(ii) the inequality (I7) has no eventually negative solutions;

(iii) all solutions of the equation (20) are oscillatory.

Proof: Let $y(t)$ be an eventually positive solution of inequality (I6). Then there exists a $t_{1} \geq t_{0}$ such that

$$
y(t)>0, y(t-\tau)>0 \text { and } y[g(t, \xi)]>0, t \geq t_{1}, \xi \in J
$$

Setting

$$
z(t)=y(t)+\lambda(t) y(t-\tau), t \geq t_{1}
$$

we obtain from (I6) that

$$
z^{\prime \prime}(t) \leq-\int_{a}^{b} P(t, \xi) F(y[g(t, \xi)]) d \sigma(\xi) \leq 0, \quad t \geq t_{1},
$$

and thus the function $z^{\prime}(t)$ is monotone decreasing in the interval $\left[t_{1},+\infty\right)$. Suppose that there exists a $t_{2} \geq t_{1}$ such that

$$
z^{\prime}\left(t_{2}\right)=\alpha<0
$$

then we have

$$
z^{\prime}(t) \leq z^{\prime}\left(t_{2}\right)=\alpha \text { for } t \geq t_{2} .
$$

Integrating both sides of the inequality (24) from $t_{2}$ to $t\left(t>t_{2}\right)$, we obtain

$$
z(t) \leq z\left(t_{2}\right)+\alpha\left(t-t_{2}\right)
$$


Hence $\limsup _{t \rightarrow+\infty} z(t) \leq 0$ which contradicts the assumption that $y(t)$ is an eventually positive solution. Thus we have

which implies

$$
z^{\prime}(t) \geq 0, t \geq t_{1}
$$

$$
z(t) \geq k_{1}>0 \text { for } t \geq t_{1}
$$

From Lemma 1 it follows that there exists a closed and measurable set $E \subset\left[t_{1},+\infty\right)$ and a constant $k>0$ such that

$$
y[g(t, \xi)] \geq k, \quad t \in E, \quad \xi \in J
$$

and

$$
\operatorname{meas}(E \cap[t, t+2 \tau]) \geq \tau, \quad t \geq t_{1} .
$$

By (21) we have

$$
F(y[g(t, \xi)]) \geq F(k)>0, t \in E, \xi \in J
$$

Integrating both sides of inequality (23), we get

$$
\begin{gathered}
F(k) \int_{E \cap\left[t_{1}, t\right]} \int_{a}^{b} P(s, \xi) d \sigma(\xi) d s \leq \int_{t_{1}}^{t} \int_{a}^{b} P(s, \xi) F(y[g(s, \xi)]) d \sigma(\xi) d s \\
\leq z^{\prime}\left(t_{1}\right)-z^{\prime}(t) \leq z^{\prime}\left(t_{1}\right) .
\end{gathered}
$$

Taking $t \rightarrow+\infty$, we have

$$
\int_{E} \int_{a}^{b} P(s, \xi) d \sigma(\xi) d s<+\infty
$$

which contradicts (22). This proves assertion (i). Assertion (ii) follows from the fact that, if $y(t)$ is an eventually negative solution of $(I 7)$, then $-y(t)$ is an eventually positive solution of (I6). The proof of assertion (iii) is obvious.

\section{Further Oscillation Criteria}

In this section, we shall establish some further oscillation criteria for problems $(E)-(B 1)$ and $(E)-(B 2)$ using the results obtained in the last two sections.

Theorem 5: Assume that condition (6) holds and $\lambda(t) \geq 0$ for $t \geq t_{0}>0$. If

and

$$
\liminf _{t \rightarrow+\infty} \int_{t_{1}}^{t}\left(1-\frac{s}{t}\right) R_{1}(s) d s=-\infty
$$




$$
\underset{t \rightarrow+\infty}{\limsup } \int_{t_{1}}^{t}\left(1-\frac{s}{t}\right) R_{1}(s) d s=+\infty
$$

for every sufficiently large $t_{1} \geq t_{0}$, then all solutions of problem $(E)-(B 1)$ are oscillatory in $G$.

Proof: Note that due to condition (26) we can see that the differential inequality (I1) has no eventually positive solutions by Theorem 3 . Since from (27) it follows that

$$
\liminf _{t \rightarrow+\infty} \int_{t_{1}}^{t}\left(1-\frac{s}{t}\right)\left[-R_{1}(s)\right] d s=-\limsup _{t \rightarrow+\infty} \int_{t_{1}}^{t}\left(1-\frac{s}{t}\right) R_{1}(s) d s=-\infty
$$

differential inequality (I2) has no eventually positive solutions by Theorem 3 either. Hence all solutions of problem $(E)-(B 1)$ are oscillatory in $G$ by Theorem 1. The proof is complete.

The following result can be proved similarly with the use of Theorem 3 and Theorem 2 .

Theorem 6: Assume that condition (6) holds and that $\lambda(t) \geq 0$ for $t \geq t_{0}>0$. If

and

$$
\liminf _{t \rightarrow+\infty} \int_{t_{1}}^{t}\left(1-\frac{s}{t}\right) R_{2}(s) d s=-\infty
$$

$$
\limsup _{t \rightarrow+\infty} \int_{t_{1}}^{t}\left(1-\frac{s}{t}\right) R_{2}(s) d s=+\infty
$$

for every sufficiently large $t_{1}>t_{0}$, then all solutions of problem $(E)-(B 2)$ are oscillatory in $G$.

A consequence of Theorem 1 and Theorem 4 in the case of $f(x, t) \equiv 0$ and $\mu(x, t) \equiv 0$ is the following result.

Theorem 7: Assume that conditions (6) and (21) hold, and that $0<\lambda_{1} \leq \lambda(t) \leq \lambda_{2}, t \geq t_{0}$. If, for any closed and measurable set $E \subset\left[t_{0},+\infty\right)$ for which meas $(E \cap[t, t+2 \tau]) \geq \tau, t \geq t_{0}$, it is true that

$$
\int_{E} \int_{a}^{b} \min _{x \in \bar{\Omega}}\{p(x, t, \xi)\} d \sigma(\xi) d t=+\infty
$$

then all solutions of $(E)$ satisfying the boundary condition

$$
\frac{\partial u}{\partial N}+\gamma(x, t) u=0,(x, t) \in \partial \Omega \times R_{+}
$$

are oscillatory in $G$.

The following result is a consequence of Theorem 2 and Theorem 4 when $f(x, t) \equiv 0$ and $\phi(x, t) \equiv 0$.

Theorem 8: If all assumptions of Theorem 7 hold, then all solutions of equation $(E)$ satisfying the boundary condition

$$
u=0, \quad(x, t) \in \partial \Omega \times R_{+}
$$

are oscillatory in $G$.

Remark: Using Theorems 1-6 in [1], we can establish some oscillation for boundary value 
problems $(E)-\left(B 1^{*}\right)$ and $(E)-\left(B 2^{*}\right)$ when $f(x, t) \equiv 0$ and the details are omitted.

To conclude this paper, we consider an example.

Example: Consider the neutral hyperbolic equation

$$
\begin{gathered}
u_{t t}+u_{t t}(x, t-\pi)+2 \int_{-\pi}^{0} e^{-t} u(x, t+\xi) d \xi=u_{x x}+e^{t} \cos x\left[\sin t+2\left(1-e^{-\pi}\right) \cos t-\left(1+e^{-\pi}\right) e^{-2 t}\right] \\
(x, t) \in\left(0, \frac{\pi}{2}\right) \times(0,+\infty)
\end{gathered}
$$

with boundary condition of the type $(B 1)$

$$
-u_{x}(0, t)=0, u_{x}\left(\frac{\pi}{2}, t\right)=-e^{t} \sin t, t \geq 0 .
$$

Here $\quad n=1, \quad \Omega=\left(0, \frac{\pi}{2}\right), \quad \lambda(t)=1, \quad p(x, t, \xi)=2 e^{-t}, \quad F(u)=u, \quad g(t, \xi)=t+\xi, \quad a(t)=1$, $f(x, t)=e^{t} \cos x\left[\sin t+2\left(1-e^{-\pi}\right) \cos t-\left(1+e^{-\pi}\right) e^{-2 t}\right], \int_{\partial \Omega} \mu(x, t) d s=-e^{t} \sin t$. It is easy to see
that

$$
\begin{gathered}
R_{1}(t)=\frac{1}{|\Omega|} \int_{\partial \Omega} \mu(x, t) d S+\frac{1}{|\Omega|} \int_{\Omega} f(x, t) d x=\frac{4}{\pi}\left(1-e^{-\pi}\right) e^{t} \cos t-\frac{2}{\pi}\left(1+e^{-\pi}\right) e^{-t}, \\
\int_{t_{1}}^{t}\left(1-\frac{s}{t}\right) R_{1}(s) d s=\int_{t_{1}}^{t}\left(1-\frac{2}{t}\right)\left[\frac{4}{\pi}\left(1-e^{-\pi}\right) e^{s} \cos s-\frac{2}{\pi}\left(1+e^{-\pi}\right) e^{-s}\right] d s \\
=\frac{2}{\pi}\left(1-e^{-\pi}\right) \frac{e^{t}}{t} \sin t-\frac{2}{\pi}\left(1+\epsilon^{-\pi}\right) \frac{1}{t e^{t}}+\frac{1}{t} C_{1}+C_{2},
\end{gathered}
$$

where $C_{1}$ and $C_{2}$ are constants which depends on $t_{1}$ only. The hypotheses of Theorem 5 are satisfied and hence every solution of problem $(28)$ and $(29)$ is oscillatory in $\left(0, \frac{\pi}{2}\right) \times(0,+\infty)$. In fact, the function $u(x, t)=e^{t} \sin t \cos x$ is such a solution.

\section{References}

[1] Liu, X.Z. and Fu, X., Nonlinear differential inequalities with distributed deviating arguments and applications, Nonlinear World 1 (1994), 409-427.

[2] Mishev, D.P., Oscillatory properties of the solutions of hyperbolic differential equations with "maximum", Hiroshima Math. J. 16 (1986), 77-83.

[3] Vladimirov, V.S., Equations of Mathematical Physics, Moscow, Nauka 1981 (in Russian).

[4] Yoshida, N., On the zeros of solutions of hyperbolic equations of neutral type, Diff. Integral Eqs. 3 (1990), 155-160.

[5] Zahariev, A.I. and Bainov, D.D., Oscillating properties of the solutions of a class of neutral type functional differential equations, Bull. Austral. Math. Soc. 22:3 (1980), 365-372. 


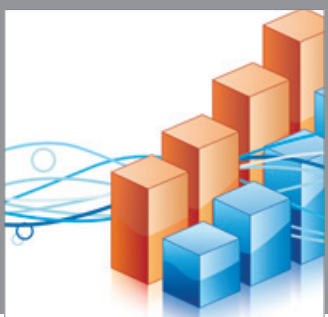

Advances in

Operations Research

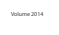

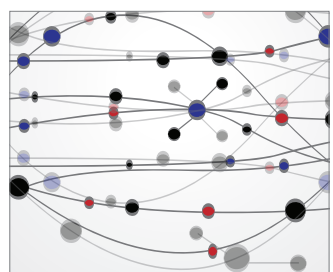

\section{The Scientific} World Journal
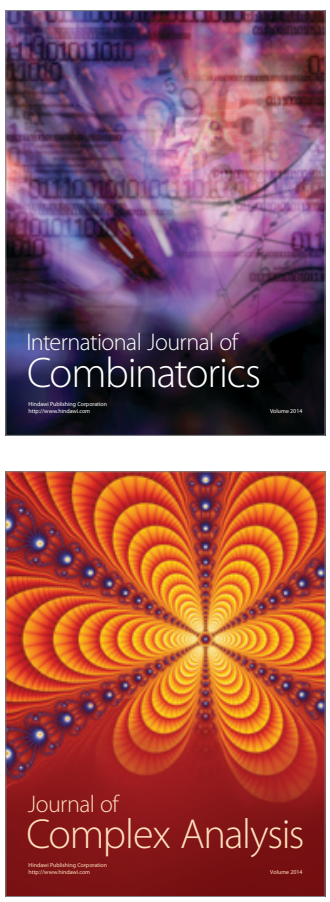

International Journal of

Mathematics and

Mathematical

Sciences
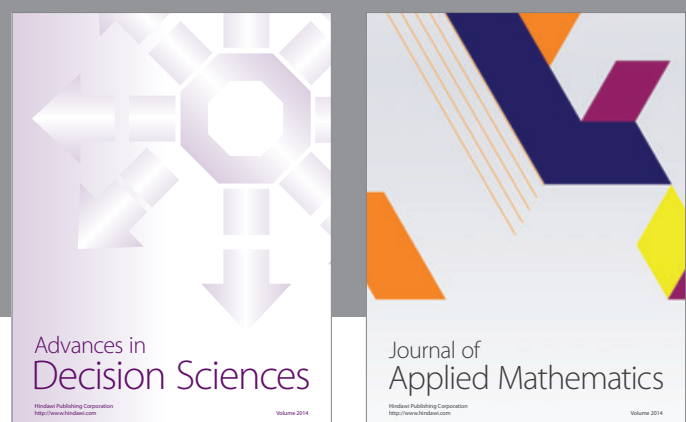

Journal of

Applied Mathematics
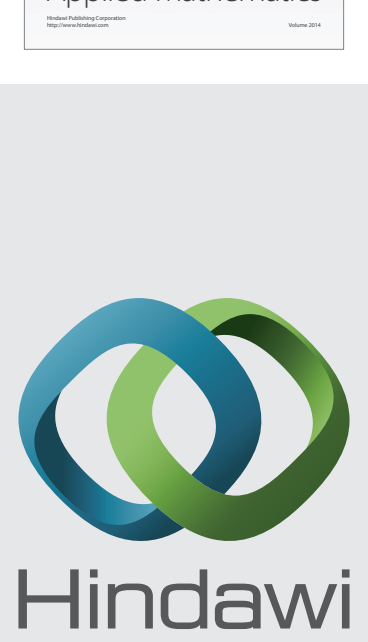

Submit your manuscripts at http://www.hindawi.com
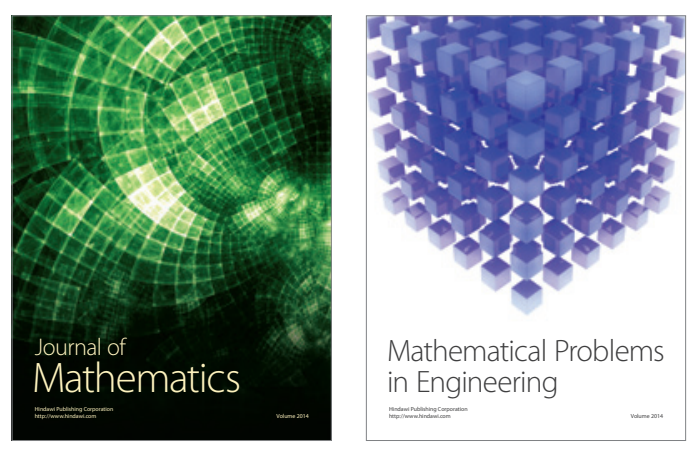

Mathematical Problems in Engineering
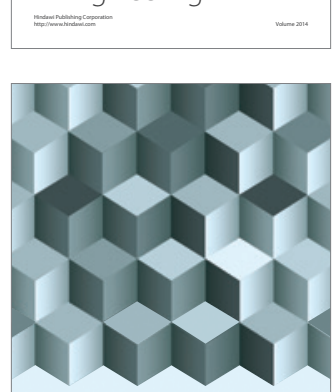

Journal of

Function Spaces
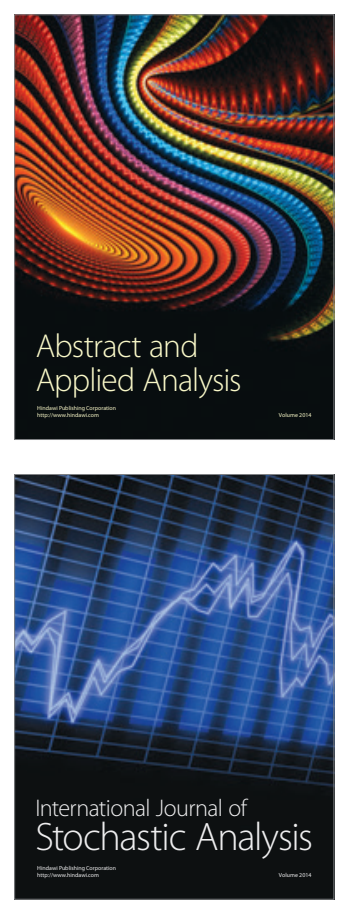

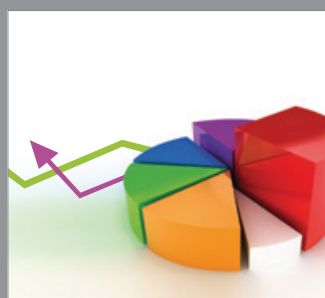

ournal of

Probability and Statistics

Promensencen
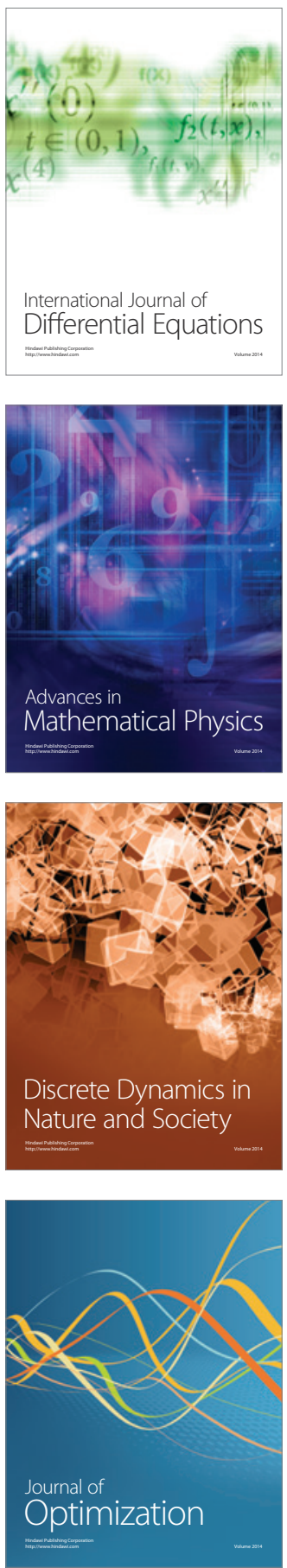